\title{
Gastrointestinal motility during sleep assessed by tracking of telemetric capsules combined with polysomnography - a pilot study
}

This article was published in the following Dove Press journal:

Clinical and Experimental Gastroenterology

9 December 2015

Number of times this article has been viewed

\author{
Anne-Mette Haase ${ }^{1}$ \\ Sibylle Fallet ${ }^{2}$ \\ Marit Otto ${ }^{3}$ \\ $S$ Mark Scott ${ }^{4}$ \\ Vincent Schlageter ${ }^{5}$ \\ Klaus Krogh' \\ 'Department of Hepatology and \\ Gastroenterology, Aarhus University \\ Hospital, Aarhus, Denmark; ${ }^{2}$ Swiss \\ Federal Institute of Technology \\ (EPFL), Lausanne, Switzerland; \\ ${ }^{3}$ Department of Neurophysiology, \\ Aarhus University Hospital, Aarhus, \\ Denmark; ${ }^{4}$ Neurogastroenterology \\ Group, Gastrointestinal Physiology \\ Unit, Queen Mary University, London, \\ UK; ${ }^{5}$ Motilis Medica SA, Lausanne, \\ Switzerland
}

\begin{abstract}
Studies of gastrointestinal function during sleep are hampered by lack of applicable techniques. Recent development of a novel ambulatory telemetric capsule system, which can be used in conjunction with polysomnography, offers a solution to this problem. The 3D-Transit system consists of ingestible electromagnetic capsules traceable through a portable extracorporeal receiver while traversing the gut. During sleep monitored by polysomnography, gastrointestinal motility was concurrently investigated using 3D-Transit in nine healthy subjects. Overall, the amplitude of gastric contractions decreased with depth of sleep (light sleep, N2 versus deep sleep, N3; $P<0.05$ ). Progression through the small intestine did not change with depth of sleep (Kruskal-Wallis probability $=0.1$ ), and there was no association between nocturnal awakenings or arousals and the occurrence of colonic or small intestinal propagating movements. Basal colonic activity was suppressed during both deep sleep $(P<0.05)$ and light sleep $(P<0.05)$ when compared with nocturnal wake periods. In conclusion, the novel ambulatory 3D-Transit system combined with polysomnography allows minimally invasive and completely ambulatory investigation of associations between sleep patterns and gastrointestinal motility.
\end{abstract}

Keywords: colonic movement, gastric contractions, sleep assessment, ingestible capsule, circadian motility, sleep stage

\section{Introduction}

Sleep has a profound inhibitory effect on gastrointestinal (GI) motility, ${ }^{1}$ with suppression of both gastric and colonic motilities clearly demonstrated in several studies. ${ }^{2-5}$ However, the lack of applicable methods and the relative inaccessibility of the GI tract have made routine investigation of whole gut motility during sleep very difficult. Nevertheless, by means of a novel telemetric capsule system ${ }^{6}-3 \mathrm{D}$-Transit system (Motilis Medica SA, Neuchatel, Switzerland) - it is now possible to investigate whole gut circadian motility. Simultaneous recordings from up to three capsules, positioned in various gut segments, make this feasible. In this pilot study, we hypothesize that by combining polysomnography and 3D-Transit, it is possible to investigate the relationship between regional GI motility patterns and sleep stages in a single examination in healthy subjects.

\section{Subjects and methods}

Sixteen healthy subjects (nine females and seven males; median age, 34 years; range, 24-52), with no history or current symptoms of GI, psychological, or sleep disorders, were included after providing informed written consent. In addition, previous major
Correspondence: Anne-Mette Haase Department of Hepatology and Gastroenterology, Aarhus University Hospital, Noerrebrogade 44, Building IC, Ist Floor, 8000 Aarhus C, Denmark

Tel +45 61658483

Fax +4578462860

Email annemette.haase@gmail.com 
abdominal surgery, medication affecting GI motility, pregnancy, and breast feeding were exclusion criteria.

Detailed information regarding GI motility profiles of these volunteers has previously been published. ${ }^{6}$

The study was conducted according to the Declaration of Helsinki and approved by the local ethics committee and the Danish Medicines Agency (reference numbers: M-20100267 and 2011123594).

\section{Methods}

\section{D-Transit}

The 3D-Transit system is a novel, ambulatory system providing whole gut transit times and various motility patterns. ${ }^{6}$ The system includes wireless telemetric capsules for ingestion, an extracorporeal receiver, and 3D-Transit software. The latter is for data analysis and real-time display (Figure 1B). The subjects wear the receiver in an abdominal belt throughout the study period (Figure 1A). Each capsule (diameter, $8 \mathrm{~mm}$; length, $21 \mathrm{~mm}$; density, $1.6 \mathrm{~g} / \mathrm{cm}^{3}$ ) incorporates a miniature battery (allowing $\sim 60$ hours recording) and an electromagnet. Following activation, the electromagnetic field continuously emitted by each capsule while traversing the gut is converted into space-time coordinates $(x ; y ; z ; \Phi ; \theta)$ using the analysis software after download. The coordinates $x ; y ; z$ express the distance (frontal, transversal, and sagittal planes) and the $\Phi ; \theta$ express the angular orientation (rotations) of each capsule with respect to the receiver (Figure 1B). Thus, changes in position, velocity, and orientation of the capsules reflect the contractile activity and progression dynamics. In order to determine capsule location, the system exploits the fact that the GI contraction frequency varies throughout the gut. The rotations of the capsule mirror the contractions of the gut. Hence, changes in the contraction frequency displayed in the raw data will, together with a matching localization of the capsule viewed in the 2D display (Figure 1B, inset), determine the shift from one GI segment to the other.

Artifacts due to breathing and posture changes are recorded by a thoracic belt and an accelerometer (Figure 1A).

The 3D-Transit system has previously been validated for the measurement of gastric emptying, small bowel, and whole gut transit. ${ }^{6,7}$ Further details on the technique and equipment have recently been published. ${ }^{6}$

\section{Polysomnography}

Sleep assessment was achieved by means of a standard technique. ${ }^{8,9}$ Polysomnography (Natus Xltec Trex ambulatory polysomnography, Type II portable device) was performed as an overnight multihour study with six electroencephalography (EEG) channels, electrooculography, electrocardiography, and chin electromyography for registration of sleep states. Airflow, oxygen saturation, respiratory effort, and body movements were not assessed since the aim of the study was not to quantify pathology.

\section{Protocol}

Preceded by an overnight fast, 16 subjects ingested two capsules (C1 at 08:00 and $\mathrm{C} 2$ at 18:30).

Meals were standardized and consumed at set times. The last meal was ingested at 18:30.

After detailed instructions from an experienced neurophysiology assistant, application of the electrodes was done just before bedtime (approximately 23:00) by a trained health care worker or by the subjects themselves. Placement was done according to the international 10-20 system and accepted guidelines. ${ }^{9}$
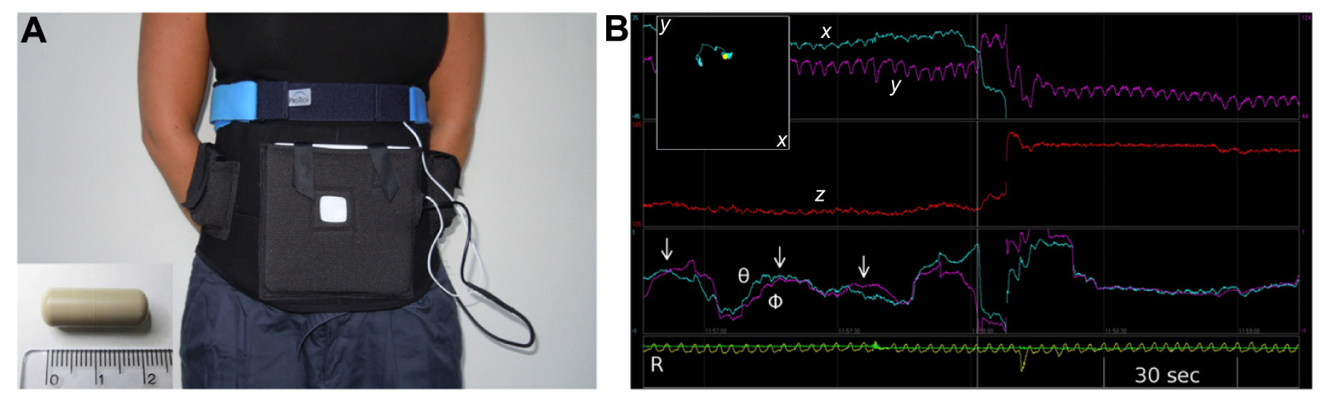

Figure I The 3D-Transit system

Notes: (A) Capsule containing an electromagnet and a battery (inset). The abdominal belt contains the 3D-Transit receiver and is worn by the subjects throughout the examination. Thoracic belt (blue) and an accelerometer inside the detector record artifacts owing to breathing and posture changes. The ruler is measuring in centimeters. (B) 3D-Transit visualization and analysis software. Changes in position and orientation of the capsules with respect to the receiver reflect gastrointestinal contractile activity and progression. Coordinates $x, y$, and $z$ display capsule movements in a frontal, sagittal, and transversal plane, respectively. The $\Phi, \theta$ coordinates describe capsule rotation, reflecting the contraction frequency (arrows mark individual contractions). The inset shows the actual movement of the capsule, which corresponds to the changes in the coordinates. The $x$-axis represents time.

Abbreviations: $\mathrm{R}$, respiration; sec, seconds. 


\section{Data analysis}

Sleep recordings (Sleep Works Natus XLTEK, Oakville, ON, Canada) were manually scored using 30 -second epochs and staged as R (rapid eye movement [REM] sleep), N1 (drowsiness), N2 (light sleep), N3 (deep sleep/slow wave sleep), or W (wake) according to the American Academy of Sleep Medicine criteria. ${ }^{9}$ Arousals, defined as changes in EEG frequency $>3$ seconds, ${ }^{9}$ were marked. EEG data artifacts were defined as technical artifacts with $50 \mathrm{~Hz}$ noise due to poor EEG electrode placement with high impedance.

Data from the polysomnography and the 3D-Transit system were synchronized based on movement artifacts in the 3D-Transit recordings and the simultaneously increased heart rate registered by the electrocardiography electrodes comprised in the polysomnography (Figure 2).

Running power spectral analysis was done to assess mean gastric contraction frequency and amplitude for each 1-minute period (MATLAB software). Speed of progression in the small intestine was also calculated for each 1-minute period. Colonic and small intestinal propagating movements (PMs) ( $\geq 3 \mathrm{~cm}$ anterograde displacement of the capsule at $\geq 1.5 \mathrm{~cm} / \mathrm{min}$ ) and basal colonic activity (mean standard deviation of the position of capsules during displacement of $<3 \mathrm{~cm}$ ) were assessed. For each subject, the aforementioned parameters were analyzed for every sleep stage and nocturnal wake periods.

All analyses were performed using STATA Version 12 (StataCorp LP, College Station, TX, USA). Data related to all study end points were nonparametric and therefore presented as median and range. Kruskal-Wallis test was performed to test whether the mean ranks were the same in the different groups. Difference between groups was tested for statistical significance with Wilcoxon rank-sum test (nonparametric test for unpaired data). Level of statistical significance was set at 0.05 .

\section{Results}

In six of the 16 experiments (38\%), missing sleep data precluded interpretation. All electrodes were correctly located on the scalp and face, but poor contact between the electrodes and the skin emerged during night, leading to artifacts. In another study, 3D-Transit failure prevented synchronization. In the remaining nine examinations, median total sleep time was 6.6 hours (range, 5.1-7.5 hours), with a median arousal index of 7.6 per hour sleep (range, 5.0-16.9 arousals/hr) and a median sleep efficiency (total sleep time/time in bed $\times 100$ ) of $79 \%$ (range, $37 \%-96 \%$ ). Median percentage of total sleep time was $4.1 \%$ in stage $\mathrm{N} 1,51.5 \%$ in stage $\mathrm{N} 2,16.7 \%$ in stage N3, and $26.4 \%$ in REM stage. Further data on sleep structure are shown in Table 1.

During sleep, $\mathrm{C} 1$ was located in the colon in all subjects. In four subjects, $\mathrm{C} 2$ was initially located in the stomach and moved to the small intestine. In five subjects, $\mathrm{C} 2$ was located in the distal part of the small intestine crossing over to the cecum during sleep.

\section{Gastric contractions}

As depicted in Table 2, gastric contraction frequency was significantly higher during nocturnal wake periods than during N2 and REM sleep. Compared with wake periods,
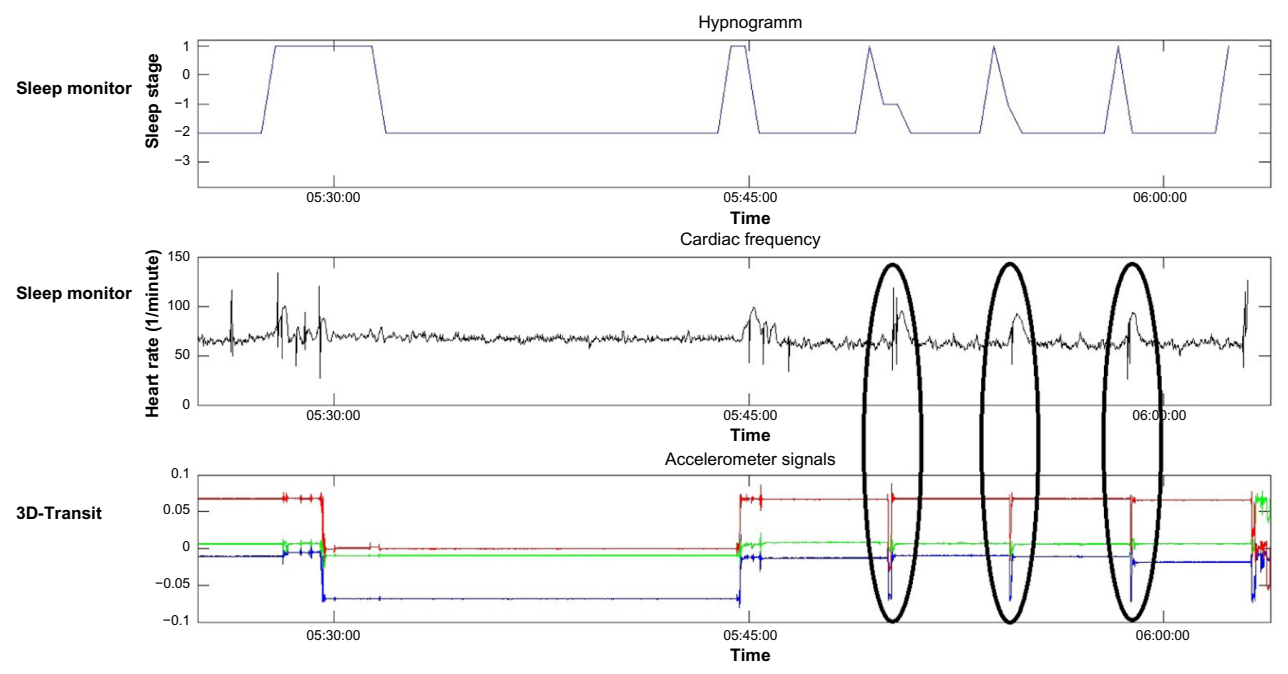

Figure 2 Data synchronization.

Notes: Posture changes obtained through the accelerometer inside the 3D-Transit receiver (red, green, and blue lines) are associated with an increased heart rate achieved through the portable sleep monitor. This way data were easily time-linked (ovals). 


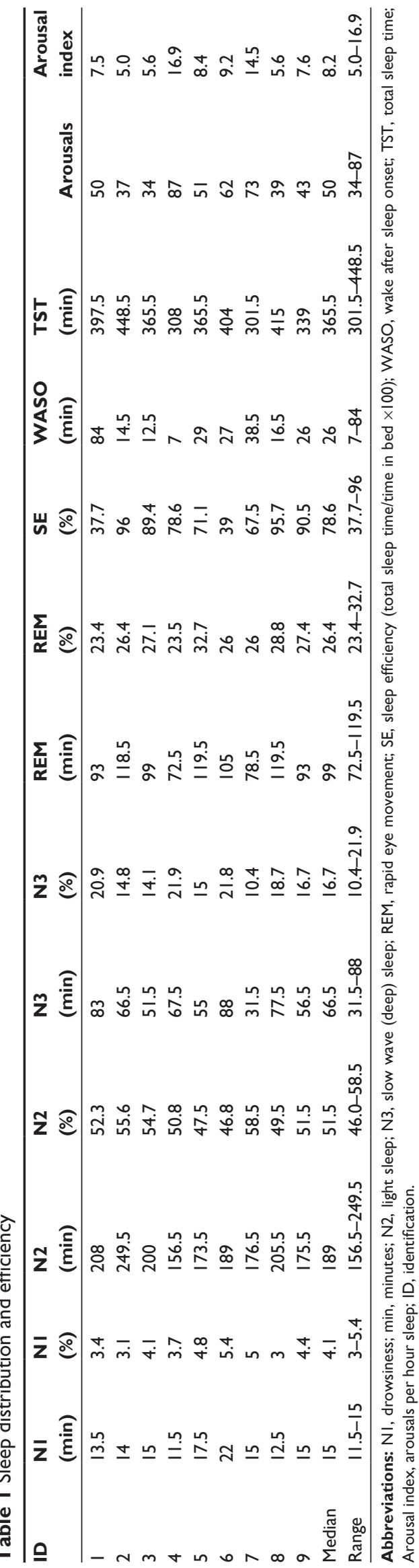

the amplitude of contractions decreased significantly during sleep. Furthermore, the amplitude during N3 sleep was significantly lower than during the other sleep stages.

\section{Progression through the Gl tract}

Progression through the small intestine did not change with depth of sleep when compared with wake periods (KruskalWallis probability $>0.05$ ). Among $136 \mathrm{PMs}$ (119 in the small intestine and 21 in the colon), 12 (9\%) occurred within 30 seconds after an arousal, 10 (7\%) during wake periods, and $114(84 \%)$ during stable sleep.

It is evident from Table 2 that basal colonic activity decreased significantly across sleep stages. Basal colonic activity was also significantly less during N3 and N2 sleep compared with wake periods.

\section{Discussion}

This pilot study has shown that simultaneous recording of whole gut GI motility and sleep states is technically possible. This holds great potential for future clinical studies exploring the relationship between sleep and GI function. By way of example, this is highly relevant in the case of inflammatory bowel disease, where a relationship between subjective sleep disturbances and disease relapse ${ }^{10}$ and subclinical inflammation appears. ${ }^{11-15}$ The method may also be useful in Parkinson's disease and irritable bowel syndrome where both GI dysmotility and sleep disorders may appear. ${ }^{16,17}$

Our results regarding sleep structure are consistent with those found in a recent population-based study. ${ }^{18}$ However, sleep efficiency tended to be lower compared with other "first night" polysomnography studies, with two of the subjects having very low sleep efficiency. A more pronounced "first night effect" due to both the polysomnography and the 3D-Transit system may have influenced the result. ${ }^{19}$ However, we believe that a full ambulatory setting, as provided by the 3D-Transit system, facilitates experiments in the home environment of the subjects, which comes closer to normal physiologic conditions than previous methods for mapping GI function employing manometry catheters or nasogastric tubes. ${ }^{2,20}$ Two consecutive nights of polysomnographic and 3D-Transit recordings may reduce the "first night effect". ${ }^{19}$

By timing capsule ingestion and meal times, it is possible to "tailor" the position of the capsules within the GI regions of interest by the time of sleep onset. ${ }^{6}$ In a previous pilot study among 20 healthy volunteers, the 3D-Transit system was found to be safe. 3D-Transit validation studies are limited by small sample sizes, and larger studies including studies on 
Table 2 Gastric contractions and basal colonic activity during sleep and nocturnal awakenings

\begin{tabular}{|c|c|c|c|c|c|c|}
\hline $\begin{array}{l}\text { Sleep } \\
\text { stage }\end{array}$ & $\begin{array}{l}\text { Gastric contractions } \\
\text { (contractions per min) } \\
\text { (median; range) }\end{array}$ & $P$-value & $\begin{array}{l}\text { Gastric contractions } \\
\text { (amplitude, } \mathrm{mm} \text { ) } \\
\text { (median; range) }\end{array}$ & $P$-value & $\begin{array}{l}\text { Colonic activity } \\
\text { (mean SD, mm) } \\
\text { (median; range) }\end{array}$ & $P$-value \\
\hline \multirow[t]{2}{*}{$\mathrm{NI}$} & $2.8(2.4-3.3)$ & NS* & $2.5(1.4-8.6)$ & $0.03 *$ & $1.40(0.5-10.4)$ & NS* \\
\hline & & & & $0.05 * *$ & & $<0.000 I^{* *}$ \\
\hline \multirow[t]{2}{*}{ N2 } & $2.6(2.2-3.8)$ & $<0.000 I^{*}$ & $2.7(0.8-9.6)$ & $0.0006 *$ & I.I5 (0.3-14.5) & $<0.000 I^{*}$ \\
\hline & & & & $<0.000 I^{* *}$ & & $<0.000 I^{* *}$ \\
\hline N3 & $2.8(2.2-3.5)$ & NS* & $2.0(0.2-8.5)$ & $<0.00 I^{*}$ & $0.88(0.3-12.1)$ & $<0.000 I^{*}$ \\
\hline \multirow[t]{2}{*}{ REM } & $2.6(2.4-3.1)$ & $<0.000$ I* $^{*}$ & $2.7(1.2-5.5)$ & $<0.000 I^{*}$ & $1.59(0.5-19.2)$ & NS* \\
\hline & & & & $0.009 * *$ & & $<0.000$ I** \\
\hline Wake & $2.9(2.2-3.4)$ & - & $5.1(1.3-9.7)$ & - & $1.69(0.5-13.5)$ & - \\
\hline
\end{tabular}

Notes: *Sleep stage compared with nocturnal wake period; **N3 compared with other sleep stages.

Abbreviations: min, minutes; NI, drowsiness; NS, not significant; N2, light sleep; N3, slow wave (deep) sleep; REM, rapid eye movement; Wake, wake periods.

different groups are needed to support our previous data. The system is, however, minimally invasive, and there is no need for bowel preparation. ${ }^{6}$ The main concern using 3D-Transit is the risk of capsule retention and thus, potentially, GI obstruction. Previous studies with capsule endoscopy have found a low risk of retention even among patients suspected of having small intestinal disease $(1.4 \%) .{ }^{21}$ The 3D-Transit capsule is smaller than the one used for capsule endoscopy, and we therefore argue that the risk of retention using the present device will be even lower.

Polysomnography is usually carried out in a sleep laboratory, but unattended monitoring as demonstrated here is possible. ${ }^{22}$ The main limitation of this study was exclusion of data from six subjects (38\%) due to artifacts caused by poor contact between the EEG electrodes and the scalp. Nevertheless, other studies using unattended polysomnography have recorded similar dropout rates. ${ }^{23}$ However, correct placement of the electrodes with impedance measurement by a sleep technologist should markedly reduce data loss in the future.

The small number of subjects recruited to the present pilot study does not allow us to draw firm conclusions about sleep and GI physiology. However, in accordance with a previous study using electrogastrography, we found that the amplitude of gastric contractions decreased with the depth of sleep. ${ }^{4}$ Yet, gastric contraction frequency did not exceed 2-4 cpm at any point in contrast to the findings of Elsenbruch et al. ${ }^{4}$

Also, in contrast to earlier studies employing small intestinal manometry, we found no change in the progression through the small intestine comparing sleep and nocturnal wake periods. ${ }^{20,24}$ This might be due to the short duration of the nocturnal wake periods. Diurnal recordings for comparison might be better for evaluating this specific end point. Future studies evaluating circadian motility patterns are required to confirm this. Also, further validation steps are required to better assess the migrating motor complex and the influence of sleep. ${ }^{20}$

Colonic motility has been shown to be generally suppressed during sleep, ${ }^{3,25,26}$ and we found increasing suppression of basal colonic activity with increasing depth of sleep and when compared to nocturnal wakening.

Contradictory results regarding the association between PMs and arousals or wake periods have previously been published. ${ }^{2,3}$ We found no association between these phenomena in this study. This could be a type II error, and larger future studies are required.

\section{Conclusion}

In conclusion, polysomnography in combination with 3D-Transit holds promise for ambulatory assessment of GI function during sleep.

\section{Author contributions}

All authors contributed toward data analysis, drafting and critically revising the paper and agree to be accountable for all aspects of the work. AMH contributed to the study design, data interpretation and analysis, preparation of the manuscript, and critical review. She is the guarantor of the article. SF contributed to the data analysis and technical review. MO contributed to the study design, data interpretation and analysis, and critical review. SMS contributed to critical review. VS contributed to technical support and technical review. KK contributed to the study design, data interpretation, preparation of manuscript, and critical review. All authors read and approved the final paper.

\section{Disclosure}

V Schlageter is a co-owner of Motilis Medica SA and a technical partner in terms of improving the system prototype. The authors report no other conflicts of interest in this work. 


\section{References}

1. Orr WC, Chen CL. Sleep and the gastrointestinal tract. Neurol Clin. 2005;23(4):1007-1024.

2. Roarty TP, Suratt PM, Hellmann P, McCallum RW. Colonic motor activity in women during sleep. Sleep. 1998;21(3):285-288.

3. Furukawa Y, Cook IJ, Panagopoulos V, McEvoy RD, Sharp DJ, Simula M. Relationship between sleep patterns and human colonic motor patterns. Gastroenterology. 1994;107(5):1372-1381.

4. Elsenbruch S, Orr WC, Harnish MJ, Chen JD. Disruption of normal gastric myoelectric functioning by sleep. Sleep. 1999;22(4):453-458.

5. Kumar D. Sleep as a modulator of human gastrointestinal motility. Gastroenterology. 1994;107(5):1548-1550.

6. Haase AM, Gregersen T, Schlageter V, et al. Pilot study trialling a new ambulatory method for the clinical assessment of regional gastrointestinal transit using multiple electromagnetic capsules. Neurogastroenterol Motil. 2014;26(12):1783-1791.

7. Worsoe J, Fynne L, Gregersen T, et al. Gastric transit and small intestinal transit time and motility assessed by a magnet tracking system. $B M C$ Gastroenterol. 2011;11(1):145.

8. Rechtschaffen A, Kales A. A Manual of Standardized Terminology, Techniques and Scoring System for Sleep Stages of Human Subjects. Bethesda, MD: US National Institute of Neurological Diseases and Blindness, Neurological Information Network; 1968. [National Institutes of Health (US) publication no 204 (Brain Information Service), University of California, Los Angeles].

9. Berry RB, Brooks R, Gamaldo CE, et al. The AASM Manual for the Scoring of Sleep and Associated Events: Rules, Terminology and Technical Specifications. Version 2.0 ed. Darien, IL: American Academy of Sleep Medicine; 2012.

10. Ananthakrishnan AN, Long MD, Martin CF, Sandler RS, Kappelman MD. Sleep disturbance and risk of active disease in patients with Crohn's disease and ulcerative colitis. Clin Gastroenterol Hepatol. 2013;11(8):965-971.

11. Kinnucan JA, Rubin DT, Ali T. Sleep and inflammatory bowel disease: exploring the relationship between sleep disturbances and inflammation. Gastroenterol Hepatol (NY). 2013;9(11):718-727.

12. Ali T, Orr WC. Sleep disturbances and inflammatory bowel disease. Inflamm Bowel Dis. 2014;20(11):1986-1995.

13. Keefer L, Stepanski EJ, Ranjbaran Z, Benson LM, Keshavarzian A. An initial report of sleep disturbance in inactive inflammatory bowel disease. J Clin Sleep Med. 2006;2(4):409-416.

14. Ranjbaran Z, Keefer L, Farhadi A, Stepanski E, Sedghi S, Keshavarzian A. Impact of sleep disturbances in inflammatory bowel disease. J Gastroenterol Hepatol. 2007;22(11):1748-1753.
15. Parekh PJ, Oldfield EC 4th, Challapallisri V, Ware JC, Johnson DA. Sleep disorders and inflammatory disease activity: chicken or the egg? Am J Gastroenterol. 2015;110(4):484-488.

16. Unger MM, Moller JC, Mankel K, et al. Patients with idiopathic rapid-eye-movement sleep behavior disorder show normal gastric motility assessed by the 13C-octanoate breath test. Mov Disord. 2011;26(14):2559-2563.

17. Chen CL, Liu TT, Yi CH, Orr WC. Evidence for altered anorectal function in irritable bowel syndrome patients with sleep disturbance. Digestion. 2011;84(3):247-251.

18. Moraes W, Piovezan R, Poyares D, Bittencourt LR, Santos-Silva R, Tufik S. Effects of aging on sleep structure throughout adulthood: a population-based study. Sleep Med. 2014;15(4):401-409.

19. Newell J, Mairesse O, Verbanck P, Neu D. Is a one-night stay in the lab really enough to conclude? First-night effect and night-to-night variability in polysomnographic recordings among different clinical population samples. Psychiatry Res. 2012;200(2-3):795-801.

20. Kumar D, Soffer EE, Wingate DL, Britto J, Das-Gupta A, Mridha K. Modulation of the duration of human postprandial motor activity by sleep. Am J Physiol. 1989;256(5 pt 1):G851-G855.

21. Li F, Gurudu SR, De Petris G, et al. Retention of the capsule endoscope: a single-center experience of 1000 capsule endoscopy procedures. Gastrointest Endosc. 2008;68(1):174-180.

22. Collop NA, Anderson WM, Boehlecke B, et al. Clinical guidelines for the use of unattended portable monitors in the diagnosis of obstructive sleep apnea in adult patients. Portable Monitoring Task Force of the American Academy of Sleep Medicine. J Clin Sleep Med. 2007;3(7):737-747.

23. Redline S, Sanders MH, Lind BK, et al. Methods for obtaining and analyzing unattended polysomnography data for a multicenter study. Sleep Heart Health Research Group. Sleep. 1998;21(7):759-767.

24. Kellow JE, Gill RC, Wingate DL. Prolonged ambulant recordings of small bowel motility demonstrate abnormalities in the irritable bowel syndrome. Gastroenterology. 1990;98(5 pt 1):1208-1218.

25. Hagger R, Kumar D, Benson M, Grundy A. Periodic colonic motor activity identified by 24 -h pancolonic ambulatory manometry in humans. Neurogastroenterol Motil. 2002;14(3):271-278.

26. Bassotti G, Germani U, Fiorella S, Roselli P, Brunori P, Whitehead WE. Intact colonic motor response to sudden awakening from sleep in patients with chronic idiopathic (slow-transit) constipation. Dis Colon Rectum. 1998;41(12):1550-1555. [discussion 1555-1556].
Clinical and Experimental Gastroenterology

\section{Publish your work in this journal}

Clinical and Experimental Gastroenterology is an international, peerreviewed, open access journal, publishing all aspects of gastroenterology in the clinic and laboratory, including: Pathology, pathophysiology of gastrointestinal disease; Investigation and treatment of gastointestinal disease; Pharmacology of drugs used in the alimentary tract;

\section{Dovepress}

Immunology/genetics/genomics related to gastrointestinal disease. This journal is indexed on CAS. The manuscript management system is completely online and includes a very quick and fair peer-review system. Visit http://www.dovepress.com/testimonials.php to read real quotes from published authors. 